\title{
The Effect of Innovative Teach-back Tool Kit Session on the Postgraduate Nursing Students' Knowledge About Injectable Artesunate
}

\author{
Sahar Ahmed", Eiman Eisa², Montaha Mohammed ${ }^{1}$, and Samah Abdalla1 \\ 'Department of Medical Nursing, Faculty of Nursing Sciences, University of Khartoum, Khartoum, \\ Sudan \\ ${ }^{2}$ Medical Surgical Nursing, Faculty of Nursing Sciences, University of Khartoum, Khartoum, Sudan \\ ORCID:
}

Sahar Ahmed Mohamed: https://orcid.org/0000-0001-7607-0679

Corresponding Author: Sahar Ahmed Mohamed; email: sa262697@gmail.com

Received 14 January 2021

Accepted 10 March 2021

Published 31 March 2021

Production and Hosting by Knowledge E

(c) Sahar Ahmed et al. This article is distributed under the terms of the Creative

Commons Attribution

which permits

unrestricted use and redistribution provided that the original author and source are credited.

Editor-in-Chief:

Prof. Mohammad A. M. Ibnouf

\section{Abstract}

Background: Injectable artesunate (inj AS) is a new anti-malarial treatment recently introduced in Sudan for the treatment of severe malaria. Inj AS has demonstrated its superiority over injectable quinine in African and Asian patients. This study aimed to evaluate the outcome of innovative teach-back tool kit session on the knowledge of inj AS among the postgraduate nursing students at the University of Khartoum, Sudan. Methods: This interventional pre-posttest studyincluded30postgraduatenursing students selected though a full-coverage sampling method and meeting the selection criteria. Data were collected using standardized self-administered questionnaire after taking a written approval from the participants. The pretest included an initial assessment followed by implementation of interactive session about new anti-malaria treatment using an innovative teach-back toolkit, and the final assessment was conducted after two weeks (posttest). The collected data were then analyzed and interpreted using descriptive and inferential statistics based on the objective and hypothesis of the study. A two-tailed $p$-value at 0.05 was considered as the level of significance using SPSS version 20.

Results: Majority of the participants were aged between 20 and 30years; of them, $26(87 \%)$ were female and $4(13 \%)$ male, and all of them had >1year of experience. The findings of the study showed that half of the participants (15[50\%]) had a good knowledge score, 12 (40\%) had a poor knowledge, and $3(10 \%)$ had a very poor knowledge score in the pretest. However, the knowledge scores increased significantly to $86.7 \%$ posttest, which is very good. The overall mean of pretest knowledge scores regarding inj AS was7.4 with a statically significant difference $(p=0.000)$. The mean score was increased to 12 with a statically significant difference posttest ( $p=0.000)$.

Conclusion: There was a statistically significant improvement in the knowledge scores of the postgraduate nursing students about inj AS after the implementation of interactive teaching session using innovative teach-back tool kit.

Keywords: inj artesunate, teach back-toolkit, nursing students, session

\section{S OPEN ACCESS}




\section{Introduction}

The World Health Organization (WHO) guidelines have recommended inj artesunate (inj AS) as the preferred medicine for treating life-threatening conditions.

Teach-back is a communication strategy in which the patients are asked to reiterate information provided to them using their own words, to confirm their understanding of the information. Teach-back isalso used as an educational approach to educate both the care givers and the patients about a new medication. The method is aproven teaching and learning strategy that improves the quality of care and ensures that healthcare professionals are educating patients and caregivers in an effective way to improve the efficacy with new medications [1].

Teach-back is a practical technique to ensure that the health professional has clearly explained a particular information to the patient and that the patient has understood what they have been told. This technique goes beyond using questions such as "Is that clear?" and "Have you understood everything?" Instead, the health professional asks the patient to explain or demonstrate, using their own words, what has just been discussed with them. Teach-back does not assess patient's knowledge but it is an effective technique to check how well the patient has understood the information provided to them by a health professional [2].

A tool kit is a special set of tools that are kept together and are often used for a particular purpose. A tool kit is the set of skills, abilities, knowledge, or other things needed in order to do a particular task or job [3]. A study conducted in the United States of America, aimed to improve patient outcomes using a tailored, evidence-based intervention to develop, encourage, and support RNs' abilities to educate and monitor patient and caregiver's knowledge of new medications early in and throughout the patient's hospitalization, used teach-back method. Results of the study showed an increase in hospital consumer assessment of healthcare provider and system (HCAHPS)scores and confirmed that teach-back is an effective method to educate patients and caregivers about new medications. Of note, since caregivers can recall the purpose and side effects of medications more often than patients, caregivers or family members should be included in patient education. As shown through this project, teach-back has been approved as a teaching and learning strategy that improves the quality of care.

Malaria is a life-threatening disease caused by parasites that are transmitted to people through the bites of infected female anopheles' mosquitoes. It is preventable and curable [4]. With an ongoing transmission of malaria in 95 countries and territories, in 2018 ,WHO reported that 219 million cases of malaria occurred worldwide, which was 
significantly lower than the 239 million cases in 2010.Malaria occurred most frequently in the WHO African region (92\%), followed by the WHO South-East Asian region (5\%) and the Eastern Mediterranean region (2\%) [5]. Approximately 300 cases of severe malaria are reported in the US each year, most of them are acquired from travel to malaria-endemic countries; cases of severe malaria should be treated with intravenous (IV)antimalarial medication. However, in the US, the Food and Drug Administration(FDA)approved IV antimalarial and IV quinine have now been discontinued, and inj AS is the first-line drug for the treatment of severe malaria [6].

Inj AS is a medication used to treat malaria and the intravenous form is preferred to quinine for severe malaria. It is often used as a part of combination therapy, such as artesunate + mefloquine, but it is not used for the prevention of malaria $[7,8]$.

Artesunate is a water-soluble hemisuccinate derivative of artemisinin indicated for the treatment of severe falciparum malaria and initiated treatment of uncomplicated falciparum malaria, when persistent vomiting precludes oral therapy. It comes in the form of powder for injection in 20,60,120, and 160-mgvial with one 1-ml ampoule of $5 \%$ sodium bicarbonate and one $5-\mathrm{ml}$ ampoule of $0.9 \%$ sodium chloride for slow IV injection (3-5 min) or slow IM injection. Nurse must dissolve the powder in the entire volume of $5 \%$ sodium bicarbonate and shake the vial until the solution becomesclear. To add the $0.9 \%$ sodium chloride into the vial, $5 \mathrm{ml}$ of the $0.9 \%$ sodium chloride is added to obtain $6 \mathrm{mlof}$ artesunate solution containing $10 \mathrm{mg} / \mathrm{ml}$ for IV injection and $2 \mathrm{ml}$ of $0.9 \%$ sodium chloride is added to obtain $3 \mathrm{ml}$ of artesunate containing $20 \mathrm{mg} / \mathrm{ml}$. The dosage and duration are as follows: childrenweighing $<20 \mathrm{~kg}: 3 \mathrm{mg} / \mathrm{kg} / \mathrm{dose}$; children weighing $\geq 20 \mathrm{~kg}$ and adult: $2.4 \mathrm{mg} / \mathrm{kg} / \mathrm{dose}$. The first dose is given on admission ( $\mathrm{HO})$, the second $12 \mathrm{hr}$ after the admission ( $\mathrm{H} 12)$, the third $24 \mathrm{hr}$ after the admission $(\mathrm{H} 24)$, and then once daily. At least three doses should be administered parenterally;if the patient can tolerate the oral route, change to an artemisinin-based combination. Of note, inj AS may cause gastrointestinal disturbances, dizziness, headache, fever, muscle and joint pain, pruritus,rashes in rare cases, QT interval prolongation, and post-treatment hemolytic anemia (especially in cases of hyperparasitemia and in young children). However, there is no contraindication for pregnancy and breast feeding [9].

In the past few years, many efficacy studies have been carried out in different regions of Sudan and the findings have shown a decreasing efficacy to artesunate + sulphadoxine-pyrimethamine (AS+SP), particularly in Gadarif State (>10\%), anda high efficacy (>95\%) of artemether-lumefantrine ( $A L)$ and dihydroartemisinin-piperaquine (DHAP).Moreover, Sudan's malaria indicators survey) MIS) 2016 showed irrational use of artemether intramuscular for treatment of uncomplicated malaria. Some published 
studies showed higher rate of non-adherence of healthcare providers to the treatment guidelines and self-treatment. In response to this situation, the malaria technical advisory committee (formed from national experts and partners) recommended the use of $A L$ as a first-line and DHAP as a second-line treatment for malaria in Sudan. The committee also recommended the use of quinine or intravenous artesunate for the treatment of severe malaria at hospital level [10].

A 2019 study in Tanzania showed the level of knowledge among healthcare providers on the preparation of inj AS in public health facilities was low [11]. Hence, this study was done to evaluate the effect of an innovative teach-back tool kit session on the postgraduate nursing students' knowledge about inj AS.

\subsection{Research questions}

1. What is the baseline knowledge of the postgraduate nursing students aboutinjectable artesunate (inj AS)?

2. What is the impact of an innovative teach-back tool kit session on postgraduate nursing students' knowledge about injectable artesunate (inj AS)?

\subsection{Research hypotheses}

HO:There will be no difference between pre- and posttest mean knowledge scores toward injectable artesunate (inj AS) using an innovative teach-back tool kit after implementation of the interactive teaching session.

$\mathrm{H1}$ : There will be a significant difference between pre- and posttest mean knowledge scores toward injectable artesunate (inj AS) using an innovative teach-back tool kit after implementation of the interactive teaching session.

\section{Materials and Methods}

\subsection{Design and setting}

An interventional pre-posttest study was conducted in the Faculty of Nursing Sciencesat University of Khartoum, Sudan. 


\subsection{Study population}

The target population of the study was students pursuing master's degree in nursing sciences in all specialties. Because the students are attached to different hospitals, they can easily transfer knowledge to their colleagues when they go back to their respective hospitals.

\subsection{Inclusion criteria}

All students pursuing the master'sdegree of nursing sciences with all specialties in batch12 in the Faculty of Nursing Sciences, University of Khartoum, Sudan.

\subsection{Exclusion criteria}

Master's students attending workshop or training on new inj AS.

\subsection{Sample size and technique}

A total of 30 students participated in the study. They were selected through a fullcoverage sampling method.

\subsection{Data collection methods}

Data were collected by the researcher using a self-administered questionnaire after conducting a pilot study to ensure the validity and reliability of the questionnaire. The questionnaire consisted of two parts: part 1 included socio demographic data and consisted of three variables (age, sex , and practical experience), part 2 consisted of knowledge regarding inj AS and included 14 close-ended questions(Yes or No). Each correct answer was given a score of " 1 " and a wrong answer was given a score of " 0 ." After that, the sum and the percentage of the total score was calculated.

\begin{tabular}{|c|c|}
\hline $11-14$ & Very good \\
\hline $7-10$ & Good \\
\hline$<6$ & Poor \\
\hline
\end{tabular}




\subsubsection{Structured interactive session}

Contents of the session:

1. Definition of teach-back.

2. Use the tool kit.

3. Definition of malaria.

4. Information about artesunate injection.

5. Therapeutic action, indications, route of administrations, dosage and duration, and contraindications of injAS.

Objective of the session

By the end of this presentation, every student will be able to:

1. Identify to the teach-back toolkit as a teaching strategy.

2. Recognize an overview about malaria and new antimalarial treatment (inj AS).

3. Should be able to calculate dose, indications, contraindications, and drug interactions.

Interactive session was conducted in 60 min and comprised of a brief talk using innovative teach-back tool kit and small group discussions.

\subsection{Data analysis}

Data were collected, coded, entered, and then analyzed using the statistical package for social sciences (SPSS) using mean, standard deviation, paired $t$-test, and $P$-value.

\section{Results}

In this interventional pre-posttest study,30 nurses were included and the collected data were tabulated, analyzed, and interpreted using description and inferential statistics based on the objective of the study. Analysis was done based on the objective and hypothesis of the study. A two-tailed $p$-value at 0.05 was considered as the level of significance. 
TABLE 1: Distribution of the study subjects according to their demographic background ( $n=30)$.

\begin{tabular}{l|l|l} 
Characteristics & Frequency & $\%$ \\
$\begin{array}{l}\text { Age }(\mathrm{yr}) \\
-20-30\end{array}$ & 28 & 93 \\
$->30$ & 2 & 7 \\
Gender & 4 & 13 \\
- Male & 26 & 87 \\
- Female & & \\
Experience $(\mathrm{yr})$ & 0 & 0 \\
$-<1$ & 30 & 100 \\
$->1$ & 30 & 100 \\
Total & &
\end{tabular}

Table 1 shows that the mean age of the participants was 25 years (age group 20 $30)$,most of the participants were female (26 [87\%]), and all participants had a nursing experience of $>1$ year.

TABLE 2: Students' knowledge about injectable artesunate (injAS) ( $n=30)$

\begin{tabular}{|c|c|c|c|c|c|c|c|c|}
\hline \multirow[t]{2}{*}{ No. } & \multirow{2}{*}{$\begin{array}{l}\text { Knowledge } \\
\text { aspects }\end{array}$} & \multicolumn{2}{|c|}{ Pretest } & \multicolumn{2}{|c|}{ Posttest } & \multicolumn{2}{|c|}{ Pretest1 and Posttest1 } & \multirow{2}{*}{$\begin{array}{c}\text { Paired } \\
\text { t-test } \\
\text { P-value }\end{array}$} \\
\hline & & Mean & SD & Mean & SD & Mean & SD & \\
\hline 1 & $\begin{array}{l}\text { Which form of } \\
\text { these drugs } \\
\text { isbetter used } \\
\text { to treat severe } \\
\text { malaria? }\end{array}$ & 1.5667 & 0.62606 & 1.2000 & 0.40684 & 0.36667 & 0.80872 & 0.019 \\
\hline 2 & $\begin{array}{l}\text { Can } \\
\text { artesunate } \\
\text { injection be } \\
\text { given IV\& IM? }\end{array}$ & 0.4333 & 0.50401 & 0.9333 & 0.25371 & -0.50000 & 0.57235 & 0.000 \\
\hline 3 & $\begin{array}{l}\text { Can } \\
\text { artesunate } \\
\text { injection be } \\
\text { given rapidly? }\end{array}$ & 0.0667 & 0.25371 & 0.1333 & 0.34575 & -0.06667 & 0.44978 & 0.423 \\
\hline 4 & $\begin{array}{l}\text { Do you know } \\
\text { how to } \\
\text { prepare } \\
\text { artesunate } \\
\text { injection? }\end{array}$ & 0.6000 & 0.49827 & 1.0000 & 0.00000 & -0.40000 & 0.49827 & 0.000 \\
\hline 5 & $\begin{array}{l}\text { Do you know } \\
\text { how to } \\
\text { calculate the } \\
\text { dose? }\end{array}$ & 0.4000 & 0.49827 & 0.9333 & 0.25371 & -0.53333 & 0.50742 & 0.000 \\
\hline 6 & $\begin{array}{l}\text { Do you know } \\
\text { different } \\
\text { formulas } \\
\text { available? }\end{array}$ & 0.5667 & 0.50401 & 0.9333 & 0.25371 & -0.36667 & 0.49013 & 0.000 \\
\hline 7 & $\begin{array}{l}\text { Do you know } \\
\text { the } \\
\text { administration } \\
\text { protocol in the } \\
\text { first } 24 \mathrm{hr} \text { ? }\end{array}$ & 0.5000 & 0.50855 & 0.9000 & -0.40000 & 0.30513 & 0.56324 & 0.001 \\
\hline
\end{tabular}




\begin{tabular}{|c|c|c|c|c|c|c|c|c|}
\hline \multirow[t]{2}{*}{ No. } & \multirow{2}{*}{$\begin{array}{l}\text { Knowledge } \\
\text { aspects }\end{array}$} & \multicolumn{2}{|c|}{ Pretest } & \multicolumn{2}{|c|}{ Posttest } & \multicolumn{2}{|c|}{ Pretest1 and Posttest1 } & \multirow{2}{*}{$\begin{array}{c}\text { Paired } \\
\text { t-test } \\
\text { P-value }\end{array}$} \\
\hline & & Mean & SD & Mean & SD & Mean & SD & \\
\hline 8 & $\begin{array}{l}\text { Do you } \\
\text { knowthe } \\
\text { administration } \\
\text { protocol after } \\
24 \text { hr? }\end{array}$ & 0.4667 & 0.50742 & 0.9000 & 0.30513 & -0.43333 & 0.56832 & 0.000 \\
\hline 9 & $\begin{array}{l}\text { Do you record } \\
\text { the dose, } \\
\text { route, ml, and } \\
\text { time given for } \\
\text { each } \\
\text { administration } \\
\text { of artesunate? }\end{array}$ & 0.6667 & 0.47946 & 0.9667 & 0.18257 & -0.30000 & 0.53498 & 0.005 \\
\hline 10 & $\begin{array}{l}\text { Do you } \\
\text { prepare a } \\
\text { fresh solution } \\
\text { for each } \\
\text { administratio- } \\
\text { nand } \\
\text { discardthe } \\
\text { unused } \\
\text { solution? }\end{array}$ & 0.5333 & 0.50742 & 1.0000 & 0.00000 & -0.46667 & 0.50742 & 0.000 \\
\hline 11 & $\begin{array}{l}\text { Is IV } \\
\text { artesunate } \\
\text { safe in } \\
\text { pregnancy } \\
\text { and breast } \\
\text { feeding? }\end{array}$ & 0.3333 & 0.47946 & 1.0000 & 0.00000 & -0.66667 & 0.47946 & 0.000 \\
\hline 12 & $\begin{array}{l}\text { Can } \\
\text { artesunatein- } \\
\text { jectioncause } \\
\text { gastrointesti- } \\
\text { nal } \\
\text { distur- } \\
\text { bance,headach } \\
\text { pruritus, QT } \\
\text { interval } \\
\text { prolongation, } \\
\text { and post } \\
\text { hemolytic } \\
\text { anemia? }\end{array}$ & 0.6000 & 0.49827 & 0.8333 & 0.37905 & -0.23333 & 0.62606 & 0.050 \\
\hline 13 & $\begin{array}{l}\text { Doesartesunat } \\
\text { withmeflo- } \\
\text { quineand } \\
\text { pyrimethamine }\end{array}$ & 0.3000 & 0.46609 & 0.7000 & 0.46609 & -0.40000 & 0.77013 & 0.008 \\
\hline 14 & $\begin{array}{l}\text { Is } \\
\text { artesunatein- } \\
\text { jectionsafe for } \\
\text { liver and } \\
\text { kidney } \\
\text { diseases? }\end{array}$ & 0.3333 & 0.47946 & 0.7000 & 0.46609 & -0.36667 & 0.55605 & 0.001 \\
\hline 15 & $\begin{array}{l}\text { Association } \\
\text { betweenpre- } \\
\text { and posttest }\end{array}$ & 7.3667 & 2.79758 & 12.1333 & 1.59164 & -4.76667 & 2.73777 & .000 \\
\hline
\end{tabular}


Table 2 shows that the knowledge of the students about inj AS was poor and that there was a considerable improvement after the implementation of the interactive teaching session.

TABLE 3: Pre-posttest association between students' demographic data and knowledge $(n=30)$.

Male
Female

$\mathbf{F}$
4
26

Mean
12.0000
12.1538

Standarddeviation

0.00000 1.71330
P-Value 0.056

Table 3 shows that there was no difference in the knowledge between male and female participants.

TABLE 4: Improvement in knowledge among participants $(n=30)$.

Score
11-14: Very good
6-10:Good
$<5:$ Poor

\begin{tabular}{|c|c|}
\hline \multicolumn{2}{|c|}{ Pretest } \\
\hline Frequency & Percentage \\
\hline 3 & 10 \\
\hline 15 & 50 \\
\hline 12 & 40 \\
\hline
\end{tabular}

\begin{tabular}{|c|c|}
\hline \multicolumn{2}{|c|}{ Posttest } \\
\hline Frequency & Percentage \\
\hline 26 & $86 ., 7$ \\
\hline 4 & 13.3 \\
\hline 0 & 0 \\
\hline
\end{tabular}

Table 3 shows the total pre- and posttest knowledge of the respondents about inj AS and the significant improvement in their knowledge after the implementation of interactive session.

\section{Discussion}

Inj AS is a new anti-malarial treatment recently introduced in Sudan. It is used to treat severe malaria. This study aimed to evaluate the effect of an innovative teach-back tool kit session on the knowledge of the students pursuing master's degree in nursing sciences about inj AS and included 30 nurses. In the pretest, an initial assessment done followed by implementation of interactive session about inj AS using an innovative teachback toolkit. A final assessment was conducted after two weeks (posttest) to evaluate the effectiveness of the interactive session.

The mean pretest knowledge regarding the question about better treatment of severe malaria was 1.6 and that of posttest was 1.2. The association between the pre- and posttest was $P=0.4$, which was not significant $(0.019>0.05)$ and the participants thought that quinine was better than artesunate as artesunate injections are unavailable in Sudan and are also expensive, as shown in Table 1. However, contrary to our results, previous studies reveled that inj AS gains advantage over other anti-malarial injections in treating severe malaria due to its tolerable side effects and high parasitic clearance. 
It is also associated with low mortality in both children and adults with fewer side effects compared to quinine [11].

However, in Tanzania mainland, inj AS is the first drug of choice while artemether injection is the alternative for treating severe malaria in the general population and quinine injection is the first-line treatment of severe malaria in pregnant women ([12]

The mean pretest knowledge about question regarding injectable artesunate given rapidly was 0.1 and that that of posttest was 0.1. The association between pre- and posttest was $P=0.1$, which was not significant (423>0.05), as shown in Table 1 .

The present study confirmed that there was a considerably low level of knowledge concerning different formulas, preparation, calculation, and administration following the protocol in pretest. This is in line with a Turkish study that found a low level of knowledge among nurses in administering IM medicines at ventrogluteal site [13]. However, the nurses' knowledge improved significantly after implementing the teaching session.

In general, the total pre- and posttest knowledge regarding inj AS is significant with the mean total of pretest 7.4 and posttest12 and the association between the total preand posttest was -4.8 which is significant $(p=0.000)$. In addition, half of the participants, that is $15,(50 \%)$ had a a good knowledge score and12 $(40 \%)$ had a poor knowledge in pretest which increased significantly to $86.7 \%$ posttest. In comparison with the study done in Tanzania which showed that the level of knowledge on the preparation of inj AS for the treatment of severe malaria among public healthcare providers in all municipalities of Dar-al-Salaam region, the majority of HCP had low knowledge (82.5\%) on how inj AS was prepared and only few had moderate (11.9\%)-to-high knowledge (5.6\%) despite its high availability in healthcare facilities and preference by HCP [11].

The present study revealed that knowledge scores of participants increased significantly after the implementation of interactive teaching session using innovative teachback tool kit.

Therefore ,nurses in clinical practice need to adopt teach-back tool kit as a teaching strategy for any new treatment. In addition, hospital policy-makers must put informational posters for any new treatment around the hospital compound which could aid in recalling the treatment protocols. In the same direction, an Italian study showed the efficacy of posters, protocols, and brochures in reducing medication errors for IV drugs administration [14].

The study reveals that even with the utility of these orientation resources, there is a high likelihood of errors due to negligence of some vital issues that might be emphasized during training. This will possibly be reflected by the fact that almost half of the HCP knew the flow pattern to follow when preparing artesunate injection and the 
difference between the IM and IV artesunate preparation but could not respond correctly as what to do when the mixture does not mix up, when to discard the prepared solution, or the correct dose by body weight [11].

\subsection{Hypothesis Testing}

HO: There will be no difference between pre- and posttest mean knowledge scores regarding the new anti malarial treatment injectable artesunate (inj AS) using an innovative teach-back tool kit.

The aforementionednull hypothesis is rejected, and the alternative hypothesis $\mathrm{HO}$ is accepted, since there was a significant differencebetween the pre- and posttest mean knowledge scores, $p<0.000$, as shown in Table 1.

$\mathrm{H1}$ : There will be a significant difference between pre- and posttest mean knowledge scores toward injectable artesunate (inj AS) using an innovative teach-back tool kit after implementation of the interactive teaching session.

Hypothesis $\mathrm{H} 1$ is accepted as there was a significant improvement in the knowledge scores of post graduate students after the administration of the interactive teaching session.

\section{Conclusion}

There was statistically significant improvement in the knowledge scores of the postgraduate nursing students about Inj AS after the implementation of interactive teaching session using the innovative teach-back tool kit.

\section{Limitations}

1. The study was limited to students pursuing master's degree in nursing sciences in the Faculty of Nursing, University of Khartoum, Sudan.

2. The sample for the study was limited to 30 students only .Future studies must be conducted in hospital settings.

3. Inadequate literature and studies to make comparisons with. 


\section{Strengths}

To the best of our knowledge, no previous study has been conducted on the current topic in Sudan.

\section{Acknowledgements}

The authors are thankful to the dean of the Faculty of Nursing Sciences and the coordinator of the master's degree of nursing sciences at the Faculty of Nursing Sciences, University of Khartoum, Sudan for their support and to the students for participating in this study.

\section{Ethical considerations}

The study protocol was approved by both the dean and the coordinator of the master's degree of nursing sciences at the Faculty of Nursing Sciences, University of Khartoum, Sudan. Also, a written agreement was signed by the participants prior to the study.

\section{Competing interests}

The authors declare that there is no conflict of interests regarding the publication of this paper.

\section{Availability of data and material}

The data used in the study are available upon reasonable request.

\section{Funding}

None.

\section{References}

[1] Prochnow, J. A., Meiers, S. J., and Scheckel, M. M. (2019). Improving patient and caregiver new medication education using an innovative teach-back toolkit. Journal of Nursing Care Quality, vol. 34, no. 2, pp. 101-106. 
[2] Scottish Health Council. (2014). The Participation Toolkit: Supporting Patient Focus and Public Involvement in NHS Scotland. Better Evaluation.

[3] Cobuild, C. (2008). Collins Cobuild Advanced Learner's English Dictionary, New Digital Edition 2008( $)$. HarperCollins Publishers.

[4] World Health Organization. (2020). Malaria: Fact Sheet. World Health Organization: Regional Office for the Eastern Mediterranean.

[5] Whitethorn, C.,Bremen, J., Daily, J., et al. (2010). Epidemiology, prevention and control of malaria in endemic areas.UpToDate.

[6] Centers forDisease Control and Prevention. (2019). Global Health-Division of Parasitic Diseases and Malaria.

[7] Centers for Disease Control and Prevention. (2016). Malaria Diagnosis \& Treatment (United States). Retrieved from: www.cdc.gov.

[8] World Health Organization. (2013). Artesunate. WHO.

[9] Drugs, E. (2013). Practical guidelines intended for physicians, pharmacists, nurses and medical auxiliaries. In Essential Drugs. Médecins sans Frontières.

[10] Federal Ministry of Health, Khartoum, Sudan. (2017).Sudan Malaria Treatment Protocol.

[11] Mikomangwa, W. P., Kaaya, C., Kilonzi, M., et al. (2019). Level of knowledge among health care providers on preparation of injectable artesunate for treatment of severe malaria in public health facilities in Tanzania. BMC Research Notes, vol. 12, no. 1, p. 224.

[12] National Environment Management Council (NEMC).(). The National Environmental Research Agenda For Tanzania.

[13] Kadam, P. and Bhalerao, S. (2010). Sample size calculation. International Journal of Ayurveda Research, vol. 1, no. 1, p. 55.

[14] Khan, A. N., Khan, M. U., Shoaib, M. H., et al. (2014). Practice nurses and pharmacists: a perspective on the expectation and experience of nurses for future collaboration. Oman Medical Journal, vol. 29, no. 4, p. 271. 